\title{
Drainless Day Case Superficial Parotidectomy with ARTISS [Solutions for Sealant]
}

\author{
Jacob Duffin, Craig McCaffer, Lakh Pabla, Shane Lester \\ ENT Department, James Cook University Hospital, Middlesbrough, UK \\ Email: jacob.duffin@nhs.net
}

How to cite this paper: Duffin, J., McCaffer, C., Pabla, L. and Lester, S. (2020) Drainless Day Case Superficial Parotidectomy with ARTISS [Solutions for Sealant]. International Journal of Otolaryngology and Head \& Neck Surgery, 9, 194-202.

https://doi.org/10.4236/ijohns.2020.96023

Received: July 15, 2020

Accepted: November 1, 2020

Published: November 4, 2020

Copyright (c) 2020 by author(s) and Scientific Research Publishing Inc. This work is licensed under the Creative Commons Attribution International License (CC BY 4.0).

http://creativecommons.org/licenses/by/4.0/

\begin{abstract}
Aims: Superficial parotidectomy (SP) is a commonly performed procedure which traditionally requires a drain and overnight hospital stay. This series aimed to show the safety and efficacy of drainless day case parotidectomy using ARTISS [Solution for Sealant] fibrin glue. Materials and Methods: Patients with a superficial parotid lump with benign preoperative sampling underwent an SP. We initially used both ARTISS and a drain, which was removed when output was $<40 \mathrm{mls}$. ARTISS without drain was then introduced and once confident patients were sent home the same day. Objectives: We prospectively collected data and divided groups into ARTISS alone and ARTISS with a drain to compare patients' length of stay and complication rates. Results: 88 patients were included; 52 ARTISS alone, 22 as a day case. $42.3 \%$ of patients where ARTISS alone was used were discharged within 24 hours, with the remainder between 24 - 48 hours. Comparatively, no ARTISS and drain patients were discharged within 24 hours and $86.1 \%$ were discharged between 24 - 48 hours with the remainder over 48 hours. There were 3 postoperative haematomas and none in the drainless group; a statistically significant difference $(p=0.034)$. Of the 6 recorded salivary leaks, 4 were in the drain group and 2 in the ARTISS alone group $(p>0.05)$. Conclusions: Comparable complication rates and reduced length of stay suggest that ARTISS in SP is safe and effective. These findings stand to benefit both patients and the NHS by improving the patient journey and reducing overall costs.
\end{abstract}

\section{Keywords}

Parotid Gland, Parotid Neoplasms, Fibrin Tissue Adhesive

\section{Introduction}

Salivary gland tumours account for 3\% of all head and neck cancer, with $70 \%$ - 
$85 \%$ of them occurring in the parotid glands [1] [2]. The majority of lesions are benign and are effectively treated by surgical excision alone [2]. The mainstay of treatment is a superficial parotidectomy, a procedure which traditionally involves the placement of a vacuum drain. This collects excess fluid, saliva and blood, preventing a collection forming under the skin. This is removed once the volumes fall below a level determined safe by the surgeon. This can take several days and is a major reason for prolonged inpatient stay.

An alternative to drain placement in superficial parotidectomy is the use of fibrin based sealants such as ARTISS [Solution for Sealant], which have been increasing in prominence in head and neck surgery [3]. ARTISS is comprised of two human plasma derived components. One is a sealer protein solution containing human fibrinogen and a synthetic fibrinolysis inhibitor (Aprotinin), which helps prevent the breakdown of the clot at the wound site [4]. The other is a low-concentration human thrombin solution in a calcium chloride solution which extends polymerisation time. Using this fibrin sealant allows the skin that is raised over the tumour to be sealed onto the surface of the remaining parotid bed, reducing dead space and preventing a potential collection accumulating under the skin. Bajwa et al. described, in a recent systematic review, that the use of fibrin sealants in soft tissue head and neck surgery is promising, though there is still a lack of sufficient evidence to support its use [5]. There have been two studies published to date which have looked at the use of ARTISS in parotidectomy and its value in reducing patient length of stay [6] [7]. Both were small studies with 34 and 31 patients recruited, which found that ARTISS reduced patient length of stay and had comparable complication rates. However, neither of these studies included information on the use of antiplatelets or anticoagulants in their patients, so it still remains unclear as to the impact of these medications on postoperative complication rates with ARTISS without drains.

This study, which is the largest of its kind, aimed to add to the body of evidence demonstrating the safety and efficacy of a drain free, day case procedure using ARTISS, including those on antiplatelets and anticoagulants, which could result in improvement in patient satisfaction, through reduction in length of patient stay and avoidance of unnecessary drains. By using King's Fund data, we also aimed to determine the degree of financial benefit which would be conferred to the NHS by reducing the length of stay.

\section{Materials and Methods}

This case series was conducted at a tertiary head and neck unit in the United Kingdom between November 2015 and December 2018, where all patients referred to ENT have their management and surgery performed by one of the two head and neck surgeons. The study included all consecutive patients over our data collection period with a superficial parotid lump who had a benign preoperative sampling (FNA/core biopsy) and underwent a superficial parotidectomy. Patients who were found to have extension to the deep lobe and required either a 
subtotal or total parotidectomy were not selected for a drainless approach.

Standard practice at our institution is to tailor resection to the extent of the lump in the form of a partial superficial parotidectomy and all lumps were excised with a cuff of normal tissue where possible to ensure adequate clearance. None of the patients underwent an extracapsular dissection.

Understanding that there may be a learning curve for the application of ARTISS, it was decided that it would be applied in addition to the normal technique where a drain is inserted. Following excision of the benign lump, a $14 \mathrm{Ch}$ Redivac vacuum drain was inserted. The wound was closed with interrupted intradermal 3 - 0 vicryl sutures, except for a short segment where the sutures were left untied, leaving a window into the surgical bed. A film of ARTISS fibrin sealant $(2 \mathrm{mls})$ was sprayed through this window over the surgical bed using a pneumatised spray applicator. The skin flap was then lowered over the surgical bed and pressure applied for 2 minutes to allow polymerisation to occur as per the manufacturer guidelines. The remaining skin edge sutures were carefully tied without pulling the flap off the parotid bed and liquiband skin glue applied to the skin. No external sutures, clips or other dressings were applied. The patients were instructed to keep the wound dry for a week then to peel the glue off at home. Prior to conducting this study, a case series looking at specific drain outputs was done which showed that $86 \%$ (23/29) of outputs were below $40 \mathrm{ml}$ by 7 am the next morning (unpublished data). Therefore, it was decided that if the drain output was less than $40 \mathrm{mls}$, the drain could be removed and the patient discharged home as per previous normal practice.

Following 25 cases in total with no significant complications the senior author, felt that the learning curve was past and moved on to using ARTISS alone, employing the same technique but without the use of a Redivac drain. Initially, patients were kept in overnight and discharged the next day as long as there were no complications. However, if the case was done at the start of an all-day list and the patient was well after 6 hours with adequate social support, they were then given the option of being discharged home the same day. This 6-hour rule was later removed and patients are now sent home the same day as long as their operations were uncomplicated and no problems were identified in the immediate postoperative period. This is now offered to all patients routinely in the absence of unavoidable circumstances, such as a lack of social support.

Each case was recorded prospectively, including patient demographics and use of antiplatelet or anticoagulant therapy. The length of stay was also noted. We retrieved notes for each patient and recorded complications retrospectively after their follow up appointment at 4 to 6 weeks. The definitive histology report was also reviewed and recorded. The sample size was determined by the number of procedures it was possible to perform within the three-year period.

Descriptive statistics were used for patient demographics and chi-squared was applied to the complication rates to determine statistical significance. A p-value of $<0.05$ was defined as statistically significant. For the purposes of statistical 
analysis, patients were divided into ARTISS and drain and ARTISS alone groups, with patients being assigned to the group postoperatively depending on whether a drain was used.

All patients included in the study were done so anonymously. As this study reflected an update in usual practice, no ethical permissions were sought.

\section{Results}

A total of 88 patients were included in the study, with 52 having ARTISS alone. The mean age in the ARTISS and drain group was 61.7 years (range $40-77$ ) and 59 years (range 18 - 81) in ARTISS alone. The ARTISS and drain group were $38.9 \%$ male and the ARTISS alone were 50.9\% male. Table 1 illustrates the percentage of patients on antiplatelet or anticoagulant therapy. Within the ARTISS alone group, 22 patients were done as day case procedures. $42.3 \%$ of the ARTISS alone group were discharged within 24 hours with the remainder between 24 48 hours. Comparatively, none of the drain group were discharged within 24 hours and $13.9 \%$ stayed longer than 48 hours.

Table 2 shows a breakdown of the complications encountered in both groups of patients for comparison. There were 3 recorded haematomas in the group with ARTISS and a drain with 2 requiring a return to theatre, compared to 0 in the ARTISS alone group, which was statistically significant $(p=0.034)$. All haematomas were identified in the immediate postoperative period. Salivary leaks were also less common in the ARTISS alone group with only 2 observed, versus 4 in the ARTISS and drain group. There was a slightly higher number of temporary, partial facial nerve weaknesses in the ARTISS alone group, however, all patients had a full recovery at their follow-up appointment.

Figure 1 and Figure 2 show a breakdown of the histology reports with pleomorphic adenomas and Warthin's making up the majority of the cases. Table 3 shows the raw figures for these data sets.

Table 1. Patient demographics and length of stay.

\begin{tabular}{ccccccccccc}
\hline & \multicolumn{2}{c}{ Age (years) } & \multicolumn{2}{c}{ Gender (\%) } & \multicolumn{2}{c}{ Anticoagulation/antiplatelet (\%) } & \multicolumn{3}{c}{ Length of stay (\%) } \\
\cline { 2 - 10 } & Mean & Male & Female & Aspirin & Clopidogrel & LMWH & $<24$ hrs & 24 - 48 hrs & $>$ 48 hrs \\
\cline { 2 - 10 } ARTISS + drain & 61.9 & 38.9 & 61.1 & 22.2 & 2.8 & 2.8 & 0 & 86.1 & 13.9 \\
ARTISS & 59 & 50.9 & 49.1 & 15.4 & 4.8 & 2.4 & 42.3 & 57.7 & 0 \\
\hline
\end{tabular}

Table 2. Comparison of raw complication figures.

\begin{tabular}{ccccccc}
\hline & \multicolumn{6}{c}{ Complications } \\
\cline { 2 - 7 } & Haematoma & $\begin{array}{c}\text { Salivary } \\
\text { leak }\end{array}$ & $\begin{array}{c}\text { Wound } \\
\text { infection }\end{array}$ & $\begin{array}{c}\text { Facial nerve } \\
\text { palsy }\end{array}$ & $\begin{array}{c}\text { First bite } \\
\text { syndrome }\end{array}$ & Total \\
\hline Artiss + drain & 3 & 4 & 4 & 4 & 1 & 16 \\
Artiss & 0 & 2 & 1 & 7 & 0 & 10 \\
$\chi^{2}$ & 0.034 & $>0.05$ & $>0.05$ & $>0.05$ & $>0.05$ & \\
\hline
\end{tabular}




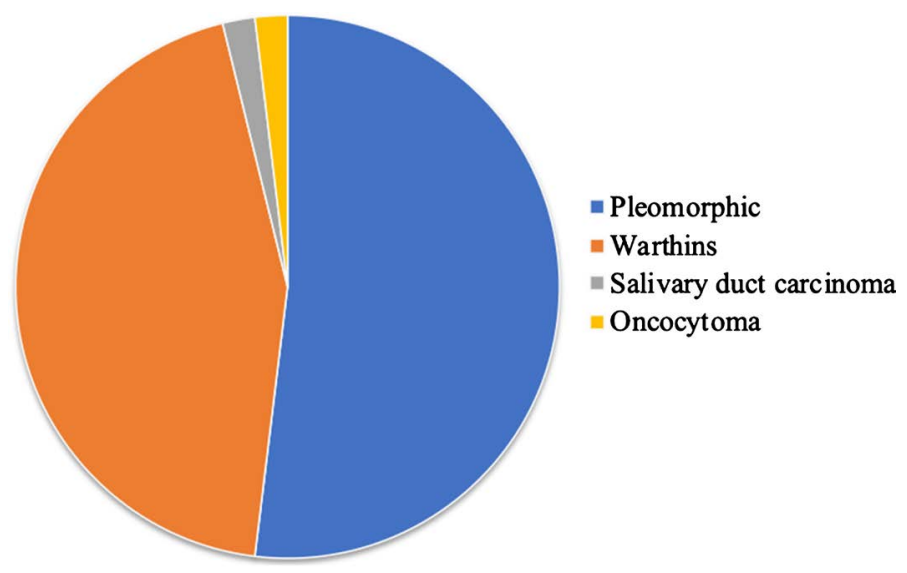

Figure 1. Histology for ARTISS group.

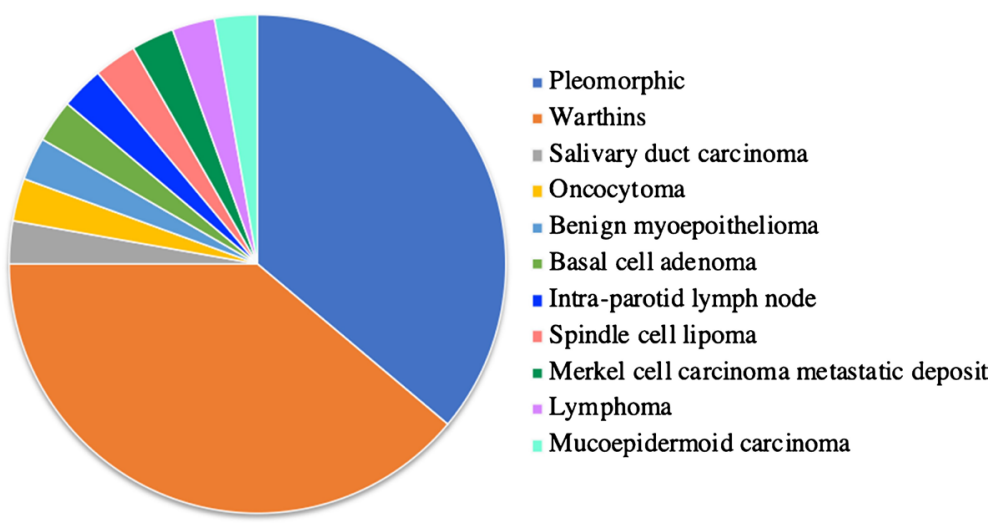

Figure 2. Histology for ARTISS and drain group.

Table 3. Raw numbers for histology of both groups.

\begin{tabular}{ccc}
\hline & Artiss + drain & Artiss \\
\hline Pleomorphic & 13 & 27 \\
Warthins & 14 & 23 \\
Oncocytoma & 1 & 1 \\
Salivary duct carcinoma & 1 & 1 \\
Benign myoepithelioma & 1 & 0 \\
Basal cell adenoma & 1 & 0 \\
Intra-parotid lymph node & 1 & 0 \\
Spindle cell lipoma & 1 & 0 \\
Merkel cell carcinoma metastatic deposit & 1 & 0 \\
Lymphoma & 1 & 0 \\
Mucoepidermoid carcinoma & 1 & 0 \\
Total & 36 & 52 \\
\hline
\end{tabular}

Surgeons found that ARTISS was easy to set up and use, with the pneumatised spray applicator allowing a suitably uniform spread of the sealant and effective closure of the wound. No immediate surgical complications were encountered 
with its use.

\section{Discussion}

In looking to streamline the patient experience and safeguard patient care with evolving technology, fibrin based sealants have emerged as a popular surgical adjunct. Their application has been noted in various specialties, particularly neurosurgery, cardiac and vascular surgery, in an attempt to reduce blood loss and provide a watertight closure of soft tissue [8] [9]. The most substantial review of fibrin sealants in head and neck surgery is the aforementioned review by Bajwa et al. which looked at the research base for its use in thyroidectomy, neck dissection, rhytidectomy and parotidectomy. The heterogeneity of studies included made the meta-analyses difficult to interpret, but the studies identified on parotidectomy showed the most promising results. A study by Maharaj et al. found that the use of Tisseel $^{\odot}$ (a similar fibrin based sealant) resulted in a statistically significant reduction in drainage output $(p<0.02)$ and a shorter average stay of 1.4 days compared to 1.6 days [3]. These findings were supported by Conboy and Brown, who in their study of 21 patients had no major complications and were performed as day cases [10]. The review identified the selectivity of patients in the studies used as being a key detractor from the strength of the results, as only the lowest risk patients were chosen. In our study we included all patients, with varying comorbid profiles, including the use of antiplatelet and anticoagulant therapy, putting them in the high risk category for postoperative bleeding. Both groups had comparable numbers on these therapies and have shown that even with the inclusion of these cases, the complication rates remain low. In addition, we demonstrated that the raw complication numbers seen with the use of ARTISS alone were better than with both ARTISS and a drain, with the difference of haematomas being statistically significant. It is appreciated that we did not have the numbers to reach statistical significance with other complication rates. Two recent studies have also looked at the use of ARTISS in performing day case parotidectomy. In Cunniffe et al., a study of 34 patients where 17 used ARTISS and 17 used drain alone, there were no haematomas, comparable numbers of salivary leaks, with 2 in the ARTISS group and 1 in the drain group and 3 versus 2 seromas in ARTISS versus drain respectively [6]. Poolovadoo et al., conducted a study of 31 patients using ARTISS with only 1 haematoma and 2 seromas [7]. These complication rates are also comparable to our own and those reported in the literature and thus day case superficial parotidectomy is a feasibly safe procedure in patients who are multi-comorbid [11].

Day case surgery in ENT is very common, with studies showing that it decreases the disruption to a patient's life and reduces the anxiety suffered by family members when their relatives are hospitalised [12]. Getting It Right First Time (GIRFT) for ENT recommended an increase in the use of day case surgery across ENT [13]. With the use of ARTISS, the introduction of day case surgery allowed $42.3 \%$ of our drainless patients to be discharged within 24 hours and as 
the learning curve continues, there is potential for this figure to increase. This supports the findings of Cunniffe et al., who found that $47 \%$ of their patients were able to go home the same day and that there was an improved mean length of stay of 0.52 days versus 1.64 days in the drain group.

There are a limited number of studies which look at the potential cost benefit associated with moving towards day case parotid surgery and none in the United Kingdom. Furthermore, Tisseel ${ }^{\circ}$ is the fibrin sealant used in all of these studies, which at a price of $\$ 167.25$ ( $£ 122.85$ ) per $2 \mathrm{mls}$, is more expensive than ARTISS $\$ 105.34$ ( $£ 77.38$ ), the fibrin sealant of choice in our study. The study done in Canada by Conboy and Brown found that the use of Tisseel ${ }^{\odot}$ in parotid surgery resulted in a saving of $\$ 1775$ CAD ( $£ 1013.17)$ per case [10]. A similar study in Spain found a saving of $€ 755.44$ ( $£ 664.26$ ), though this was in thyroid surgery [5]. Due to a lack of itemised billing in the NHS, calculating the exact costs incurred during a patient's stay is difficult. However, with the use of National Reference costs, the King's Fund estimates that the average day case cost stands at

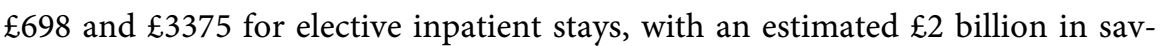
ings between 2013 and 2015 with the move towards more day case procedures [14]. According to the Hospital Episode Statistics data report 2017-18, 2490 completed treatment episodes were classified as "benign neoplasm: parotid gland' and though cases should always be judged on an individual basis, it is clear that an increase in the number of day case procedures with ARTISS would result in significant financial savings to the order of $£ 6.5$ million per year in the NHS [15]. It is also worth noting that the cost of ARTISS is also reclaimable by the trust through the purchase of blood products, extending further savings to the trust themselves.

\section{Limitations}

Although this is the largest study of its kind to date, we appreciate the limitations of what is still a small data set performed by only two surgeons and therefore we do not have the numbers to reach statistical significance on all complication rates. A well designed multi-centre randomised controlled trial would be beneficial in further determining the efficacy of ARTISS in parotidectomy. There was some heterogeneity in the types of tumours removed in the ARTISS and drain group which may have had an impact on the tendency for the surgical bed to bleed postoperatively. There was also no control of the antiplatelet and anticoagulation status of patients who underwent surgery, other than the standard preoperative considerations. Therefore, a controlled study looking at anticoagulated versus non-anticoagulated patients and patients with and without antiplatelets would provide a more substantial basis for assessment of their effects on use of fibrin based sealants in parotidectomy.

\section{Conclusion}

This case series demonstrates encouraging results for day case parotid surgery 
and provides a basis for further trials looking at performing day case parotidectomies in comorbid patients. Comparable complication rates and shorter hospital stays will improve the patient experience and overall satisfaction of the service, while making considerable savings for the NHS.

\section{Conflicts of Interest}

The authors declare no conflicts of interest regarding the publication of this paper.

\section{References}

[1] Albergotti, W., Nguyen, S., Zenk, J., et al. (2012) Extracapsular Dissection for Benign Parotid Tumors: A Meta-Analysis. Laryngoscope, 122, 1954-1960. https://doi.org/10.1002/lary.23396

[2] Huang, G., Yan, G., Wei, X., et al. (2014) Superficial Parotidectomy versus Partial Superficial Parotidectomy in Treating Benign Parotid Tumors. Oncology Letters, 9, 887-890. https://doi.org/10.3892/ol.2014.2743

[3] Maharaj, M., Diamond, C., Williams, D., et al. (2006) Tisseel to Reduce Postparotidectomy Wound Drainage: Randomized, Prospective, Controlled Trial. Journal of Otolaryngology, 35, 36-39. https://doi.org/10.2310/7070.2005.4032

[4] ARTISS [Solutions for Sealant] (2011) Summary of Product Characteristics. Baxter International Inc., Vienna.

[5] Bajwa, M., Tudur-Smith, C., Shaw, R., et al. (2017) Fibrin Sealants in Soft Tissue Surgery of the Head and Neck: A Systematic Review and Meta-Analysis of Randomised Controlled Trials. Clinical Otolaryngology, 42, 1141-1152.

https://doi.org/10.1111/coa.12837

[6] Cunniffe, H., Wong, B., Hilger, A., et al. (2019) Drain-Free Parotidectomy: A Pilot Study Using ARTISS Fibrin Sealant. European Archives of Oto-Rhino-Laryngology, 276, 2025-2029. https://doi.org/10.1007/s00405-019-05449-x

[7] Poolovadoo, Y., Aggarwal, R. and Loughran, S. (2019) The Use of ARTISS ${ }^{\mathrm{TM}}$ in Performing Parotidectomies as Day Case Surgery: A Single Centre Review of 31 Cases. Clinical Otolaryngology, 44, 847-850. https://doi.org/10.1111/coa.13386

[8] Esposito, F., Angileri, F., Kruse, P., et al. (2016) Fibrin Sealants in Dura Sealing: A Systematic Literature Review. PLoS ONE, 11, e0151533.

https://doi.org/10.1371/journal.pone.0151533

[9] Daud, S., Kaur, B., McClure, G., et al. (2020) Fibrin and Thrombin Sealants in Vascular and Cardiac Surgery: A Systematic Review and Meta-Analysis. European Journal of Vascular and Endovascular Surgery, 60, 469-478.

[10] Conboy, P. and Brown, D. (2008) Use of Tissue Sealant for Day Surgery Parotidectomy. Otolaryngology-Head and Neck Surgery, 37, 208-211.

[11] Guntinas-Lichius, O., Klussmann, J., Wittekindt, C., et al. (2006) Parotidectomy for Benign Parotid Disease at a University Teaching Hospital: Outcome of 963 Operations. Laryngoscope, 116, 534-540.

https://doi.org/10.1097/01.mlg.0000200741.37460.ea

[12] Mallick, A. (2006) Experiences of Day Surgery in Otolaryngology. Indian Journal of Otolaryngology and Head \& Neck Surgery, 58, 38-40.

[13] Marshall, A. (2019) Ear, Nose and Throat Surgery. GIRFT Programme National Specialty Report.

[14] Appleby, J. (2018) Day Case Surgery: A Good News Story for the NHS [Internet]. 
The King's Fund.

https://www.kingsfund.org.uk/blog/2015/07/day-case-surgery-good-news-story-nhs

[15] NHS Digital (2017) Hospital Admitted Patient Care Activity. NHS Digital.

https://digital.nhs.uk/data-and-information/publications/statistical/hospital-admitt ed-patient-care-activity/2017-18\#related-links 